\title{
The innate immune sensor NLRC3 attenuates Toll-like receptor signaling via modification of the signaling adaptor TRAF6 and transcription factor NF-kB
}

\author{
Monika Schneider ${ }^{1}$, Albert G Zimmermann ${ }^{2}$, Reid A Roberts ${ }^{1}$, Lu Zhang ${ }^{3}$, Karen V \\ Swanson ${ }^{2}$, Haitao Wen ${ }^{2}$, Beckley K Davis ${ }^{2,6}$, Irving C Allen ${ }^{2}$, Eda K Holl ${ }^{1,6}$, Zhengmao \\ $\mathrm{Ye}^{1,6}$, Adeeb H Rahman ${ }^{2,6}$, Brian J Conti ${ }^{1,6}$, Timothy K Eitas ${ }^{2}$, Beverly H Koller ${ }^{3}$, and Jenny \\ P-Y Ting ${ }^{1,2,4,5}$ \\ ${ }^{1}$ Department of Microbiology and Immunology, University of North Carolina at Chapel Hill, Chapel \\ Hill, North Carolina, USA \\ ${ }^{2}$ Lineberger Comprehensive Cancer Center, University of North Carolina at Chapel Hill, Chapel \\ Hill, North Carolina, USA \\ ${ }^{3}$ School of Dentistry, University of North Carolina at Chapel Hill, Chapel Hill, North Carolina, USA \\ ${ }^{4}$ Center for Translational Immunology and Institute for Inflammatory Diseases, University of North \\ Carolina at Chapel Hill, Chapel Hill, North Carolina, USA \\ ${ }^{5}$ Department of Genetics, University of North Carolina at Chapel Hill, Chapel Hill, North Carolina, \\ USA
}

\begin{abstract}
Several members of the NLR family of sensors activate innate immunity. In contrast, we found here that NLRC3 inhibited Toll-like receptor (TLR)-dependent activation of the transcription factor NF- $\kappa$ B by interacting with the TLR signaling adaptor TRAF6 to attenuate Lys63 (K63)linked ubiquitination of TRAF6 and activation of NF- $\mathrm{kB}$. We used bioinformatics to predict interactions between NLR and TRAF proteins, including interactions of TRAF with NLRC3. In vivo, macrophage expression of NIrc3 mRNA was diminished by the administration of lipopolysaccharide (LPS) but was restored when cellular activation subsided. To assess biologic relevance, we generated $\mathrm{NIrC}^{-1-}$ mice. LPS-treated $\mathrm{NIrC}^{--}$macrophages had more K63ubiquitinated TRAF6, nuclear NF- $\mathrm{kB}$ and proinflammatory cytokines. Finally, LPS-treated NIrc $3^{-1-}$ mice had more signs of inflammation. Thus, signaling via NLRC3 and TLR constitutes a
\end{abstract}

(C) 2012 Nature America, Inc. All rights reserved.

Correspondence should be addressed to J.P.-Y.T. (jenny_ting@med.unc.edu).

6 Present addresses: Department of Biology, Franklin and Marshall College, Lancaster, Pennsylvania, USA (B.K.D.); Duke

Translational Research Institute, Duke University, Durham, North Carolina, USA (E.K.H.); HIV Drug Discovery Unit, Infectious

Diseases Medicines Discovery \& Development, GlaxoSmithKline, Research Triangle Park, North Carolina, USA (Z.Y.); Department of Medicine, Division of Liver Diseases, Mount Sinai School of Medicine, New York, New York, USA (A.H.R.); and Department of Biochemistry and Molecular Biology, Oregon Health \& Science University, Portland, Oregon, USA (B.J.C.).

Note: Supplementary information is available in the online version of the paper.

\section{AUTHOR CONTRIBUTIONS}

M.S. did most of the experiments; J.P.-Y.T. supervised the project; M.S. and J.P.-Y.T. prepared the manuscript; A.G.Z. and B.H.K. generated the NIrc $3^{-1}$ mouse; L.Z., H.W. and T.K.E. contributed to biochemical analysis; K.V.S. did confocal assays; B.K.D. helped in design and creation of NLRC3 site mutants; I.C.A. contributed to experimental design and execution of mouse studies; B.J.C. designed NLRC3 retroviral vectors; R.A.R. did bioinformatics analysis and contributed to biochemical analysis; and A.H.R., E.K.H. and Z.Y. contributed to characterization of the $\mathrm{NIrC}^{-1-}$ mouse.

COMPETING FINANCIAL INTERESTS

The authors declare no competing financial interests. 
negative feedback loop. Furthermore, prevalent NLR-TRAF interactions suggest the formation of a 'TRAFasome' complex.

The NLR (nucleotide-binding, leucine-rich repeat-containing) family of sensors has many functions, including inflammatory and anti-inflammatory roles. This group is defined by a conserved structure composed of a nucleotide-binding domain (NBD), a variable number of leucine-rich repeats and an amino-terminal effector domain, commonly a pyrin domain or a caspase-recruitment domain ${ }^{1}$. The most commonly studied subgroup of NLR proteins is composed of the inflammasome-forming NLR proteins. NLRP1, NLRP2, NLRP3, NLRC4, NLRC5, NLRP6 and NLRP7 are each able to form an inflammasome complex, which leads to the processing and activation of pro-caspase- 1 to caspase- 1 , all of which can occur with or without the adaptor ASC (PYCARD), depending on the NLR and the experimental conditions $^{1-6}$. The inflammasome responds to a wide array of pathogen- or dangerassociated molecular patterns. Pro-interleukin $1 \beta$ (pro-IL-1 $\beta$ ) accumulates after a primary inflammatory signal, and this accrual is frequently the result of activation of NF- $\kappa \mathrm{B}$. This first signal, which often relies on the activation of NF- $\kappa \mathrm{B}$, can also modulate NLR expression $^{7}$.

Although most inflammasome NLR proteins do not alter NF- $\kappa$ B signaling, a small number of NLR proteins can positively modulate NF- $\kappa \mathrm{B}$ activity after specific stimuli. For example, the receptors Nod1 and Nod2 are associated with activation of NF- $\kappa \mathrm{B}$ after stimulation with peptidoglycan ${ }^{8-11}$. Both Nod1 and Nod2 associate with the adaptor RIP2, which can lead to its Lys63 (K63)-linked ubiquitination by the E3 ubiquitin ligases cIAP1 and cIAP2 and enhancement of the activation of NF- $\kappa B^{12-15}$. Although Nod1 and Nod2 are associated with the activation of NF- $\kappa \mathrm{B}$, NLR proteins are also able to attenuate inflammatory pathways. NLRP12 inhibits the canonical NF- $\kappa$ B signaling pathway by preventing the accumulation of hyperphosphorylated kinase IRAK1 and noncanonical signaling via NF- $\kappa$ B through association with and degradation of NIK, a kinase necessary for phosphorylation of the serine-threonine kinase IKKa and downstream processing of the NF- $\mathrm{\kappa B}$ precursor $\mathrm{p} 100$ to the p52 NF- $\kappa$ B subunit ${ }^{16}$. Nlrp12-null mice have more activation of canonical and noncanonical NF- $\kappa$ B in models of colitis and colon cancer ${ }^{17,18}$. NLRX1 and NLRC5 have been described as having inhibitory effects on proinflammatory signaling. NLRX1 can inhibit the type I interferon pathway by interfering with the adaptor MAVS-RNA helicase RIG-I pathway and the NF- $\kappa$ B pathway via the signaling adaptor TRAF6 (refs. 19-21), and NLRC5 inhibits type I interferon and NF- $\kappa B^{22,23}$. However, there is controversy about the role of NLRC5 as an activator or inhibitor of those pathways both in cell lines and in Nlrc $^{-1-}$ mice $^{24-26}$.

Activation of NF- $\mathrm{\kappa B}$ is a key regulator of inflammation and can be promoted through various stimuli, which results in the production of a plethora of proinflammatory cytokines, including tumor-necrosis factor (TNF), IL-1 $\beta$ and IL-6. NF- $\kappa$ B can be stimulated through the receptor for IL-1 or Toll-like receptors (TLRs) to activate the IKK complex, which leads to the translocation of heterodimers of the NF- $\kappa$ B subunits p65 and p50 to the nucleus ${ }^{27-30}$. Myd88 and TRIF are adaptors required for the recruitment of IRAK1, IRAK4 and TRAF6, which leads to activation of the kinases TAK1 and IKK. Protein modifications such as ubiquitination of IRAK1 and TRAF6 (refs. 31, 32) and phosphorylation of TAK1 and IKK ${ }^{33}$ are essential for this pathway ${ }^{34,35}$. TRAF proteins are key components of activation of the immune system. During activation, TRAF molecules autoubiquitinate through the E3 ubiquitin ligase in their RING domain, except for TRAF1, which lacks a RING domain ${ }^{36}$. K48-linked ubiquitin typically is associated with degradation via the $26 \mathrm{~S}$ proteosome. K63linked ubiquitin is nondegradative. It can cause a conformational change, act as a scaffold or change localization of the substrate and cause activation of TRAF6 (refs. 37, 38). Because 
ubiquitination of TRAF6 is a key regulatory event, it is often a target for regulation by inhibitors of NF- $\mathrm{kB}$, such as A20 and CYLD ${ }^{39-43}$.

NLRC3 (CLR16.2) is an understudied NLR, and its function has been examined in only one study, to our knowledge, through the use of overexpression systems in cell lines in which NF- $\kappa B$ activation was diminished in the presence of overexpressed NLRC3 (ref. 44). Here we describe an inhibitory mechanism used by NLRC 3 to regulate NF- $\kappa B$ through interaction with TRAF6 and to alter the ubiquitination state of TRAF6. Studies of $\mathrm{NIrC3}^{-/-}$mice confirmed the inhibition of proinflammatory signaling, K63-linked ubiquitination of TRAF6 and nuclear translocation of NF- $\mathrm{xB}$ by NLRC3. NLRC3 therefore serves as a checkpoint to prevent a dysregulated inflammatory response.

\section{RESULTS}

\section{NLRC3 inhibits MyD88-dependent activation of NF-KB}

NLRC 3 is expressed mainly by cells of the immune response ${ }^{44}$, so to better understand its function, we focused on the innate immune pathway, specifically TLR signaling pathways. TLRs and MyD88 are important for the detection of bacterial and viral pathogen-associated molecular patterns and are often the first molecules to respond to infection. To initially assess the function of NLRC3, we transfected human embryonic kidney (HEK293T) cells with an NF- $\mathrm{kB}$ luciferase reporter vector, an activating protein and increasing concentrations of NLRC3. NLRC3 significantly inhibited NF- $\times B$ activation in a dosedependent manner in response to an activating signal from MyD88 and TRAF6 and, to a lesser but not significant extent, through IRAK, but not through IKKa or p65 (Fig. 1a). These results suggested that NLRC3 was able to interfere with upstream signaling via MyD88 and/or TRAF6 but not downstream activation by IKKa or $\mathrm{p} 65$.

As myeloid-monocytic cells are the main cell types that engage in TLR signaling, we assessed the ability of NLRC3 to inhibit MyD88-dependent activation of NF- $\mathrm{kB}$ in the human monocyte-like THP-1 cell line. We transduced THP-1 cells with recombinant retrovirus expressing a stable short hairpin RNA that targets mRNA encoding NLRC3 (shNLRC3) or overexpressing a vector encoding NLRC3. We found substantial expression of NLRC3 in human monocytic cell lines (THP-1 and U937) and of Nlrc3 in mouse primary bone marrow macrophages (Supplementary Fig. 1a). We confirmed by real-time PCR the overexpression of NLRC3 and lower expression of mRNA encoding NLRC3 achieved through the use of shNLRC3 (Supplementary Fig. 1b). To determine if NLRC3 was involved in TLR signaling, we stimulated those THP-1 lines with lipopolysaccharide (LPS). Overexpression of NLRC 3 resulted in less TNF and IL6 mRNA, whereas shNLRC3 resulted in more $T N F$ and $I L 6 \mathrm{mRNA}$ (Fig. 1b, c). In addition, the secretion of TNF and IL-6 was also inversely associated with the expression of $N L R C 3 \mathrm{mRNA}$ (Fig. 1d, e). These findings suggested that NLRC3 may be a negative regulator of NF- $\kappa$ B signaling in monocytes.

The transcription of genes encoding cytokines occurs after MyD88-TRAF6 signaling, followed by activation of the IKK complex and phosphorylation of the NF- $\mathrm{KB}$ inhibitor $\mathrm{I} \kappa \mathrm{Ba}$. This post-translational modification leads to the ubiquitination and subsequent degradation of I $\mathrm{Ba}$. To assess if the differences observed in cytokine production were due to changes in I $\kappa \mathrm{Ba}$ modification, we assessed total and phosphorylated I $\kappa \mathrm{B} a$ by immunoblot analysis. In cells overexpressing NLRC3, phosphorylation of I $\mathrm{KBa}$ was slightly delayed (Fig. 1f). Conversely, cells with shNLRC3 had early phosphorylation of IкBa (Fig. 1f). Together these results indicated that NLRC3 diminished LPS-dependent NF- $\mathrm{BB}$ signaling in a human monocytic cell line. 


\section{NLRC3 inhibits NF-kB by association with TRAF6}

TRAF molecules are important intermediaries of the activation of NF- $\kappa B$. Overexpression of NLRC 3 was sufficient to inhibit the activation of NF- $\kappa$ B by TRAF6. To determine if NLRC3 was able to interact with TRAF6, we transiently transfected HEK293T cells to express V5-tagged TRAF6 (V5-TRAF6) and hemagglutinin-tagged NLRC3 (HA-NLRC3) and coimmunoprecipitated proteins from the cells; we found that NLRC3 associated with TRAF6 (Fig. 2a). Because of the nature of overexpression systems and the lack of a highquality antibody for the detection of endogenous NLRC3, we were unable to confirm that this interaction occurred with physiological amounts of NLRC3 or TRAF6. However, a search for TRAF-binding domains in NLRC3 showed that human NLRC3 has two major TRAF2-binding motifs ((P/S/A/T)-X-(Q/E)-E, where 'P/S/A/T' indicates proline, serine, alanine or threonine, ' $\mathrm{X}$ ' indicates any amino acid, and ' $\mathrm{Q} / \mathrm{E}$ ' indicates glutamine or glutamic acid) in its NBD (Fig. 2b). However, it has been noted that the TRAF2-binding domain is not specific to TRAF2 but is indicative of a general TRAF-binding motif ${ }^{45}$. For example, Nod2 has a predicted TRAF2-binding motif in its nucleotide-binding oligomerization domain, which facilitates association with TRAF4 (ref. 46). We found that the region in NLRC3 with the TRAF motif is conserved across various species (Fig. 2c). Because of homology in the NLR family, we searched the sequences of other NLR proteins for TRAF-binding motifs. We found that most NLR proteins have a major TRAF2-binding sequence, a minor TRAF2-binding motif and/or a TRAF6-binding motif, as described before $^{47}$ (Table 1). These data indicated that interactions between NLR and TRAF molecules may be prevalent and identified previously unknown potential intersections of NLR proteins with TRAF proteins.

We next assessed the possibility that NLRC3 associates with TRAF6 through that TRAFbinding motif in the NBD. We substituted alanine for all four amino acids in either the TRAF-binding site with the sequence Ser-Leu-Gln-Glu (site 1) or the TRAF-binding site with the sequence Ser-Val-Glu-Glu (site 2). We expressed those NLRC3 mutants together with V5-TRAF6 in HEK293T cells and assessed the association of TRAF6 and NLRC3 by immunoprecipitation of V5-TRAF6 and immunoblot analysis of HA-NLRC3. The substitution in site 1 of NLRC 3 resulted in much less association, whereas the substitution in site 2 had less or little effect (Fig. 2d). These findings indicated that the first TRAF-binding motif in the NBD of NLRC3 was important for its association with TRAF6.

\section{NLRC3 influences the degradation and ubiquitination of TRAF6}

We next explored the mechanism by which NLRC3 affected the function of TRAF6. When we transiently transfected HEK293T cells to express V5-TRAF6 and/or HA-NLRC3, we consistently noted less V5-TRAF6 in the presence of HA-NLRC3, whereas the amount of TRAF2 or TRAF3 (control proteins) remained unchanged (Fig. 3a). Additionally, the NLRC3-dependent decrease in TRAF6 protein was dose dependent (Fig. 3b). Proteins are frequently targeted to the proteosome for degradation by conjugation with K48-linked ubiquitin chains, whereas K63-linked ubiquitin is a common activating signal. Other NLR proteins that regulate NF- $\kappa \mathrm{B}$, such as Nod1 and Nod2, do so through interactions with ubiquitinated substrates, such as RIP2 (refs. 13, 14). To determine if NLRC3 affected the ubiquitination of TRAF molecules, we transfected HEK293T cells to express Myc-tagged TRAF2 or TRAF6, as well as hemagglutinin-tagged ubiquitin, in the presence or absence of vector encoding Flag-tagged NLRC3. We lysed cells in a stringent 1\% SDS buffer and boiled them so that only covalent modifications remained. We immunoprecipitated TRAF proteins and quantified ubiquitin by densitometry. The presence of NLRC3 did not significantly affect the amount of ubiquitin on TRAF2 (Fig. 3c, d) but did result in less ubiquitination of TRAF6 (Fig. 3c, d). To determine the type of ubiquitin on TRAF6 that was affected by NLRC3, we transfected HEK293T cells to express TRAF6 with NLRC3 in the 
presence of plasmid encoding total ubiquitin, K48-linked ubiquitin or K63-linked ubiquitin. The presence of NLRC3 resulted in less total ubiquitin on TRAF6, accompanied by less K63-linked ubiquitin, but there was no significant change in K48-linked ubiquitin (Fig. 3e, f). Although there was an apparent trend by which the presence of NLRC3 resulted in more K48-linked ubiquitination of TRAF6, the difference was variable and was not significant. This indicated that NLRC3 diminished the activating K63-linked ubiquitination of TRAF6 but left unchanged or enhanced the degradative K48-linked ubiquitination. The finding that K48-linked ubiquitin on TRAF6 remained in the presence of NLRC3 was consistent with the overall lower abundance of TRAF6 in the presence of NLRC3. Additionally, if NLRC3 prevented the accumulation of K63-linked ubiquitin on TRAF6 and left only K48-linked ubiquitin, then TRAF6 may have not only remained inactive but also may have been increasingly targeted to the proteosome. Because tight regulation of TRAF6 is important for NF- $\mathrm{kB}$ signaling, NLRC3 may inhibit proinflammatory signaling through this modification.

\section{More proinflammatory cytokines made by $\mathrm{NIrC3}^{-1-}$ macrophages}

The studies reported above relied on overexpressed proteins, which are useful for determining mechanistic events but can provide results that are inconsistent with endogenous processes. Therefore, to address the physiological role of NLRC3 in vivo, we generated NIrC3-deficient $\left(\mathrm{NIrC}^{--}{ }^{-}\right)$mice by homologous recombination (Fig. $4 \mathrm{a}$ ). The NBD, which is encoded by exon 3, is important for NLR protein function; therefore, we designed a targeting vector that, after homologous recombination with the endogenous locus, removed exon 3, replacing it with the gene encoding resistance to the aminoglycoside antibiotic geneticin. We used embryonic stem cells from 129S6 mice that expressed the properly modified locus to generate chimeric mice. We bred male chimeras with female C57BL/6 mice and identified germline transmission in the offspring by coat color and confirmed this by Southern blot analysis (Fig. 4b). We bred those offspring to C57BL/6J mice. We intercrossed heterozygous $\left(\mathrm{NlrC}^{+-}\right)$mice for nine generations to generate the F9 $\mathrm{NICC}^{-1-}$ mice used in these studies. Mendelian ratios for each genotype and the distribution of the targeted allele among male and female mice were normal. We verified genotype by PCR and determined absence of expression by RT-PCR (Supplementary Fig. 2a). Nlrc $3^{-1-}$ mice did not seem to have any anatomic abnormalities, similar to mice lacking other NLR proteins. Reproductive performance, gender distribution, frequency of lymphocytes and monocytes in the spleen, and total number of splenocytes, peritoneal cells and bone marrow cells were equivalent to those of wild-type mice (Supplementary Fig. 2b-e).

As NLRC3 resulted in less production of proinflammatory cytokines in human cell lines (Fig. 1), we sought to assess if a change in cytokines also occurred in NIrc $3^{-1}$ macrophages treated with LPS and whether this change was due to a greater abundance of transcripts encoding proinflammatory cytokines. The cytokine-encoding genes $T n f, I 11 b, I 16$ and $I 112$ are targets for activation by NF- $\mathrm{kB}$, and expression of these genes increases considerably after stimulation of the TLR. To determine if $\mathrm{NIrC}^{-/-}$macrophages had higher expression of genes encoding proinflammatory cytokines, we measured transcripts by real-time PCR. There was much higher expression of those cytokine-encoding genes in $\mathrm{NIrC}^{-1-}$ peritoneal macrophages than in wild-type cells after $1 \mathrm{~h}$ of stimulation with LPS (Fig. 4c). However, there was no difference between wild-type and $\mathrm{Nlrc}^{-/-}$cells in expression of the gene encoding interferon- $\beta$ after stimulation with LPS (Fig. 4d). At later time points, the expression of Tnf, IIIb and II6 in Nlrc $3^{--}$cells was similar to that in wildtype cells. Differences in the expression of TLR or TLR-signaling molecules could lead to variation in cytokine release. However, the expression of Tlr4 and Traf6 in peritoneal macrophages was similar in wild-type and NIrc $3^{-1-}$ cells (Fig. 4e). Additionally, a negative feedback loop can be activated through NF- $\kappa$ B, by which expression of NF- $\kappa$ B inhibitors such as A20, CYLD and $\mathrm{I} \kappa \mathrm{Ba}$ is increased. To assess this possible repressive effect, we measured expression of 
the genes encoding A20 (Tnfaip3; called ' $A 20$ here), CYLD (Cyld) and IkBa (Nfkbia) before and after stimulation with LPS. We observed no difference between wild-type and $\mathrm{Nlrc}^{-1-}$ macrophages in their expression of Cyld or Nfkbia before or after stimulation (Fig. 4f). In contrast, $A 20$ expression was modestly higher in $N 1 r 3^{-1-}$ macrophages than in wildtype macrophages at $1 \mathrm{~h}$ after stimulation with LPS and was much higher in $\mathrm{NIrC} 3^{-/-}$ macrophages than in wild-type macrophages by $6 \mathrm{~h}$ after treatment with LPS (Fig. 4f). That finding was in agreement with published reports indicating a negative regulatory loop between activation of NF- $\mathrm{KB}$ and $A 20$ expression ${ }^{48}$. These data indicated that the absence of NLRC3 resulted in an early increase in the transcription of NF- $\kappa B$-dependent genes activated by TLR4. That change was not preceded by a change in the expression of Tlr4, Traf6, Cyld or Nfkbia (Fig. 4d-f).

Peritoneal macrophages from NIrc ${ }^{-1-}$ mice also secreted more TNF and IL-6 than did wildtype macrophages in response to TLR agonists that activate signaling via the MyD88dependent pathway (the synthetic lipopeptide $\mathrm{Pam}_{3}$ Cys for TLR2; LPS for TLR4; and single-stranded RNA for TLR7 and TLR8) but not to a significant extent in response to an agonist that activates signaling via the TRIF-dependent pathway (the synthetic RNA duplex poly(I:C) for TLR3; Fig. 5a). Additionally, quantification of TRAF6 protein in wild-type and $\mathrm{Nlrc}^{-1-}$ peritoneal macrophages showed that cells lacking NLRC3 had more TRAF6 (Fig. 5b). Bone marrow-derived macrophages also had more TRAF6, although this greater abundance was not as substantial and varied by experiment (data not shown). That observation supported the earlier finding obtained with HEK293T cells showing that overexpression of NLRC3 led to less TRAF6 protein. To determine if NIrc3 expression was affected by stimulation of the TLR, we measured Nlrc3 expression in macrophages after in vitro stimulation with LPS. Expression diminished soon after stimulation and remained low throughout the assay interval, although by the end of the study, there was a modest recovery (Fig. 5c). As the macrophage culture began to lose viability at those later time points, the lack of recovery of NIrc3 expression might have been to due to the unhealthy state of the culture. To overcome the artifacts of tissue culture, we examined the in vivo effect of LPS on NIrC3 expression in peritoneal cells isolated at various time points after intraperitoneal injection of LPS. NIrc3 expression decreased after stimulation and this decrease was sustained for $48 \mathrm{~h}$, but by $108 \mathrm{~h}, N I r c 3$ expression recovered to the amount before stimulation (Fig. 5d). That finding suggested that the decrease in NIrc3 expression during LPS treatment was probably necessary for the inflammatory response to proceed, whereas its recovery later restored the basal resting state (Fig. 5d). Thus, regulation of NIrc3 mRNA expression provides a mechanism by which NLRC3 function is controlled.

Because there was less TRAF6 protein in the presence of NLRC3, whereas LPS diminished NIrc3 expression, we assessed the effect of NLRC3 on TRAF6 during stimulation with LPS. We measured TRAF6 protein in wild-type and $\mathrm{NIrC}^{-/-}$peritoneal macrophages before and after stimulation with LPS. In basal conditions, $\mathrm{NIrC3}^{-1-}$ macrophages had much more TRAF6 than did wild-type cells. However, at $24 \mathrm{~h}$ after LPS stimulation, when NIrc3 expression was low in wild-type cells, wild-type and $\mathrm{NIrC}^{-1-}$ cells had an equal amount of TRAF6 (Fig. 5e).

\section{More activation of TRAF6 and NF-KB in $\mathrm{NIrC3}^{-/-}$macrophages}

To better determine the function of NLRC3 in NF- $\mathrm{KB}$ signaling, we explored the mechanism by which NLRC3 inhibited the macrophage proinflammatory response. Because we had observed less ubiquitinated TRAF6 in the presence of NLRC 3 when those proteins were overexpressed, we assessed if the deletion of Nlrc3 resulted in more ubiquitination of TRAF6. To measure ubiquitination, we activated TRAF6 by timed stimulation of bone marrow-derived macrophages with LPS. We immunoprecipitated endogenous TRAF6 and measured K63-linked ubiquitin associated with TRAF6 through the use of an antibody 
specific for K63-linked ubiquitin chains. We did not study K48-linked ubiquitin because antibodies to K48-linked ubiquitin did not work properly in our experiments. We used stringent lysis conditions to disrupt any noncovalent bonds. There was more K63-linked ubiquitin on TRAF6 from NIrc ${ }^{-/-}$cells than on TRAF6 from wild-type control cells at all time points, and possibly even in the absence of stimulation (Fig. 6a). These data suggested that NLRC3 negatively regulated K63-linked ubiquitination of TRAF6, which would be expected to diminish downstream signaling. To determine if the absence of NLRC 3 led to more NF- $\kappa \mathrm{B}$ signaling, we assessed the nuclear localization of $\mathrm{p} 65$ and the abundance of p65 and I $\kappa \mathrm{Ba}$. After stimulation with LPS, the nuclei of $\mathrm{NIrC}^{-1-}$ macrophages had much more p65 than did those of wild-type cells (Fig. 6b); nearly $70 \%$ more nuclei contained p65 in $\mathrm{NIC}^{-/-}$macrophages than in wild-type cells (Fig. 6b). Additionally, there was earlier and more-prolonged phosphorylation of p65 in NIrc $3^{-1-}$ macrophages than in wild-type cells (Fig. 6c). That greater phosphorylation correlated with more-rapid loss of total IxBa and lack of recovery of I $\mathrm{KBa}$ at $60 \mathrm{~min}$ (Fig. 6d). TRAF6 can affect other proinflammatory pathways, notably the mitogen-activated protein kinase signaling pathway. To determine if the effect of NLRC 3 on TRAF6 was specific for the activation of NF- $\kappa B$, we examined phosphorylation of the mitogen-activated protein kinases p38, Erk and Jnk after stimulation with LPS. Signaling via this pathway was not affected much in the absence of NLRC3 (Fig. 6e).

\section{Enhanced response of $\mathrm{NIrC3}^{-/-}$mice to endotoxic shock}

The experiments reported above indicated that NLRC3 functioned as an attenuator of the response to LPS in macrophages. We next assessed its role as an in vivo regulator of the severe inflammatory condition of endotoxic shock. LPS is a common component of Gramnegative bacteria and induces the production of proinflammatory cytokines. An uncontrolled immune response to LPS can lead to a cytokine storm and serves as a surrogate model of sepsis. The NF- $\kappa$ B pathway is a chief driver of this inflammation. Because of the enhanced release of proinflammatory cytokines in response to TLR agonists in both human and mouse cells lacking NLRC3, we hypothesized that $\mathrm{NIrC}^{-/-}$mice would be more susceptible to LPS-induced shock. To assess this, we injected wild-type and $\mathrm{NIrC}^{-1-}$ mice with LPS or PBS (vehicle control) and monitored temperature and weight. In response to a nonlethal dose of LPS, wild-type and $\mathrm{NICC}^{-1-}$ mice initially had a similar drop in temperature and weight. However, Nlrc $^{-1-}$ mice had a significant delay in recovery, as measured by their lower body temperature and weight after challenge with LPS (Fig. 7a, b).

The body temperatures of wild-type and NIrc $3^{-1-}$ mice began to diverge $18-42 \mathrm{~h}$ after LPS challenge, with $\mathrm{NIrC}^{-/-}$mice showing a greater drop than that of control mice. Similarly, NIrc $3^{-I-}$ mice lost more weight than control mice did from $42 \mathrm{~h}$ to $88 \mathrm{~h}$ after LPS stimulation. To determine a possible cause for the diminished health of $\mathrm{NIrC}^{-1-}$ mice, we killed the mice at $24 \mathrm{~h}$ after the LPS challenge. Because we injected the mice intraperitoneally, we collected peritoneal lavage fluid to assess not only cellularity due to inflammatory infiltrates but also local cytokine concentrations, and we collected serum to assess systemic cytokine concentrations. The serum of $\mathrm{NIrc}^{-1-}$ mice had a significantly higher concentration of IL- 6 at $24 \mathrm{~h}$ after LPS treatment than did wild-type serum; additionally, the concentration of TNF was also higher in NIrc $3^{-1-}$ serum than in wild-type serum (Fig. 7c). We noted a much greater abundance of $I 16$ transcripts and IL-6 protein in the peritoneal cavity of $\mathrm{NIrC}^{-1-}$ mice than in that of wild-type mice at $24 \mathrm{~h}$ after LPS challenge (Fig. 7d, e). By $48 \mathrm{~h}$, the concentration of IL-6 was lower in both wild-type and NIrc $3^{--}$mice (Fig. 7c, d). Additionally, NIrc $3^{-/-}$peritoneal cells had higher expression of genes encoding the proinflammatory cytokines IL- 6 and TNF than did wild-type peritoneal cells (Fig. 7e). The absence of NLRC3 affected TNF less than it affected IL-6 and did not have a significant effect on IL-1 $\beta$ (Fig. 7c-e). As $\mathrm{NIrc}^{-/-}$peritoneal macrophages produced 
more proinflammatory cytokines ex vivo, it is possible that the enhancement in systemic cytokines was due to changes in cellular composition. To assess this, we examined the cellular composition of the peritoneal cavity of wild-type and $\mathrm{NIrC}^{-1-}$ mice after challenge with LPS. Although there was not a significant difference between the mice in the total proportion of cells in the peritoneal cavity, there was slightly greater frequency of peritoneal monocytes in $\mathrm{NIrC}^{-/-}$mice than in wild-type mice (Fig. 7f). However, this modest difference was probably not responsible for greater differences in the abundance of $I I 6$ transcripts and IL-6 protein. Together these results indicated that NLRC 3 had a protective role in endotoxic shock by diminishing proinflammatory cytokines, especially IL-6 and, to a lesser extent, TNF. In contrast to inflammasome NLR proteins, NLRC3 had little effect on IL-1 $\beta$. The lack of an effect on IL- $1 \beta$ might have accounted for the relatively modest effect of NLRC 3 on body temperature during exposure to endotoxins.

\section{DISCUSSION}

Although it has long been accepted that Nod1 and Nod 2 are important for the activation of $\mathrm{NF}-\kappa \mathrm{B}$, NLR proteins that negatively affect this pathway and regulate inflammatory responses have not been widely documented because of the lack of in vivo evidence ${ }^{16}$. In many cases, a complete mechanism has not been fully described and the inhibitory effect on NF- $\kappa B$ is partial ${ }^{20,21,23,24}$. Our study here has provided evidence that NLRC3 negatively affected the activation of NF- $\mathrm{KB}$ and has suggested that multiple NLR proteins may serve as regulatory checkpoints for inflammation. So far, physiological roles for inhibitory NLR proteins have been described for NLRX1 and NLRP12, whereby NLRX1 interacts with TRAF6 and diminishes its function in transformed cells, and NLRP12 promotes the degradation of NIK possibly through interactions with TRAF3 (refs. 17, 20, 21). However, the mechanism by which NLRX1 affects TRAF6 function and how NLRP12 affects TRAF3 remain elusive. It will be useful to understand the mechanisms that govern these interactions because the ability to target one molecule without disrupting all processes is ideal for the development of disease-directed therapy.

Our study has provided in vivo evidence that NLRC3 functioned as an attenuating checkpoint for the production of inflammatory cytokines in macrophages. NLRC3 diminished the production of inflammatory cytokines in vivo and improved health in an endotoxic shock model. Furthermore, our study has provided a mechanism whereby NLRC3 negatively affected endotoxin signaling by associating with TRAF6, which led to several effects on TRAF6. These effects include a lower abundance of TRAF6 protein and less activation of TRAF6 by K63-linked ubiquitination, followed by less activation and nuclear translocation NF- $\mathrm{KB}$. Our results have suggested a negative feedback loop whereby TLR signaling resulted in lower expression of NLRC3, which thus 'released' the checkpoint and permitted activation of NF- $\kappa$ B to proceed. Control of NF- $\kappa$ B through a decrease in the ubiquitination of TRAF6 is a critical step in ensuring TLR responses are not dysregulated and involves other checkpoint molecules, including A20 and CYLD ${ }^{49}$. Our work has described a regulatory function for NLRC 3 and has emphasized the need for multiple and precise tunings of TRAF6 signaling.

The bioinformatics data presented here have identified many putative interaction sites between NLR proteins and TRAF proteins. Published studies have confirmed some of these interactions and have demonstrated that they are functionally revelant ${ }^{20,21,46}$. A diverse set of signaling pathways is mediated by the TRAF molecules, including those that control proliferation, survival and inflammation. Given the range of functions of the NLR proteins that have been characterized and the number of NLR proteins that are yet to be mechanistically defined, we speculate that the functional and biochemical interactions of NLR proteins and TRAF proteins may compose an intricate checks-and-balances system of 
control, and we propose that these complexes be called 'TRAFasomes'. In addition, our study has shown that TLR signaling resulted in lower expression of NLRC3, which reflected a feedback loop between NLR proteins and TLRs.

As a working model, we hypothesize that under resting conditions, NLRC3 associated with TRAF6 and prevented activation by inhibiting the ability of TRAF6 to autoubiquitinate. After stimulation, repression by NLRC3 was released and proinflammatory signaling was promoted partly through lower expression of the gene encoding NLRC3. In the absence of NLRC3, inflammation was accelerated, which indicated that NLRC3 represents a checkpoint for TLR-induced inflammation. Expression of the gene encoding NLRC3 was lowered by LPS, but then it began to recover slowly after the inflammatory response subsided. This supported the hypothesis that NLRC3 prevents basal signaling rather than acting as an inducible inhibitor. In sum, our report has identified an NLR protein that affected TRAF6 ubiquitination as a mechanism for altering TLR signaling, whereas TLR signaling lowered NIrc3 expression to allow activation of the immune system.

\section{ONLINE METHODS}

\section{Cell culture, transfection, coimmunoprecipitation and immunoblot analysis}

HEK293T cells and peritoneal macrophages were grown in DMEM supplemented with 10\% FBS and penicillin-streptomycin. Resident peritoneal macrophages were removed by lavage and cultured overnight and nonadherent cells were removed, followed by replacement with fresh medium. Bone marrow-derived macrophages were cultured for $6 \mathrm{~d}$ in DMEM supplemented with 10\% FBS, L-glutamine, penicillin-streptomycin and $20 \%$ conditioned medium from L929 mouse fibroblasts. Transfections were done with FuGENE ${ }^{6}$ according to the manufacturer's protocol (Roche). Plasmids encoding the following were used: Myctagged TRAF6, V5-TRAF6, HA-NLRC3 (ref. 45), NF- kB-luciferase reporter and p65. NLRC3 site mutants were made by standard molecular biology subcloning techniques. Hemagglutinin-tagged MyD88 and Flag-tagged IRAK were provided by S. Ghosh; plasmids encoding hemagglutinin-tagged K48-linked ubiquitin and K63-linked ubiquitin were obtained from Y. Xiong; and Flag-tagged IKKa was obtained from A. Baldwin. Cells were lysed in 1\% Triton X-100 isotonic buffer and coimmunoprecipitation was done with V5agarose beads (Sigma) or $1 \mu \mathrm{g}$ anti-TRAF6 (D-10; Santa Cruz) and UltraLink Protein A/G beads (Pierce). Immunoblots were probed with antibody to Flag-tagged horseradish peroxidase (M2; Sigma), antibody to V5-tagged horseradish peroxidase (R961-25; Invitrogen), anti-TRAF6 (D-10 or H-274; Santa Cruz), antibody to phosphorylated IkBa (2859S; Cell Signaling Technology), anti-IxBa (4812; Cell Signaling Technology), antibody to K63-linked ubiquitin (BML-PW0600; Enzo Lifesciences), anti-actin-horseradish peroxidase (C-1 1; Santa Cruz).

\section{Stable overexpressing and knockdown THP-1 lines}

Short hairpin RNA cassettes were made with a small interfering expression cassette kit from Ambion $\left(5^{\prime}-\right.$

CGGCGAAGCTTTTTCCAAAAAAGGGAACACGACTTCCACACATCTCTTGAATGT GTGAAGTCGTGTTCCCCGGTGTTTCGTCCTTTCCACAAG-3'). These constructs were subcloned into the pHSPG vector via EcoRV and XbaI. The pHSPG-Flag-tagged NLRC3 plasmid was created by PCR amplification of sequence encoding NLRC3 with an aminoterminal Flag tag and subcloning into pHSPG via 5' XhoI-3' XhoI. Recombinant retroviruses were created by transient transfection of human A293T human embryonic kidney cells and THP- 1 cells were transduced with virus as described ${ }^{50}$. Cells were sorted for expression of green fluorescent protein and were cultured in supplemented RPMI-1640 medium with $10 \%$ FBS. 


\section{Luciferase assay}

HEK293T cells were transfected as described above with $150 \mathrm{ng}$ NF- $\mathrm{kB}$-luciferase; 0,100 or $250 \mathrm{ng}$ NLRC3; 0,150 or $250 \mathrm{ng}$ pcDNA3.1 (empty vector); and $250 \mathrm{ng}$ vector encoding MyD88, IRAK, TRAF6, IKKa or p65. Then, $24 \mathrm{~h}$ after transfection, cells were lysed in Reporter Assay Buffer (Roche). Plates were 'read' on a luminometer with the addition of ATP buffer (100 $\mu \mathrm{M}$ ATP, glycine-glycine, $\mathrm{MgSO}_{4}, \mathrm{KHPO}_{4}$, EGTA and DTT) and luciferin buffer.

\section{Ubiquitination assay}

For in vitro ubiquitination assays, HEK293T cells were transfected and then lysed $24 \mathrm{~h}$ later in $1 \%$ SDS isotonic buffer. Lysates were boiled for $15 \mathrm{~min}$ for removal of any noncovalent interactions, then were centrifuged for $10 \mathrm{~min}$ at 16,000 r.p.m., then supernatants were transferred to a new tube. Lysates were diluted with $1 \%$ Triton X-100 buffer and substrate molecules were immunoprecipitated with Myc or V5 agarose gel (Sigma).

\section{Real-time PCR}

RNA was extracted from THP-1 cells or peritoneal macrophages with the RNeasy Mini Kit according to the manufacturer's protocol (Qiagen). The cDNA was synthesized with total RNA, Superscript III (Invitrogen) and RNase inhibitors (Roche) according to the manufacturer's protocol (Invitrogen). The expression of human and mouse genes encoding TNF, IL-6, IL-1b, TNF, IL-12, TLR4, A20, CYLD, interferon- $\beta$, I $\kappa$ B $\alpha$, TRAF6 and GAPDH was assessed by real-time PCR with mouse Taqman Gene Expression Assays (Applied Biosystems); results were normalized to expression of the gene encoding GAPDH and were quantified by the change-in-threshold method $\left(\Delta \Delta \mathrm{C}_{\mathrm{T}}\right)$.

\section{Confocal microscopy}

Macrophages $\left(4 \times 10^{5}\right)$ were seeded onto coverslips in 24-well dishes and were grown for $24 \mathrm{~h}$ before stimulation with $1 \mu \mathrm{g} / \mathrm{ml}$ LPS. Cells were fixed with $4 \%$ paraformaldehyde, followed by ice-cold methanol. Cells were stained with anti-p65 (D14E12; Cell Signaling) and Alexa Fluor 546-conjugated antibody to rabbit (A-11035; Invitrogen) and then were counterstained for nucleic acids with Hoechst 33342. Cells were analyzed with a Zeiss LSM 710 laser-scanning confocal microscope.

\section{Enzyme-linked immunosorbent assay}

Peritoneal lavage fluid, serum and cell culture supernatants were collected and assayed for cytokines. Cytokine production was measured by enzyme-linked immunosorbent assay of human (eBioscience) or mouse (BD Bioscience) TNF, IL-1 $\beta$ and IL-6 according to the manufacturer's protocol.

\section{Generation of $\mathrm{NIrC3}^{-1-}$ mice and in vivo LPS treatment}

The 3' arm of homology of the NIrc3-deficient construct was obtained by PCR (Takara LA Taq) through the use of mouse 129/Sv genomic DNA with a forward primer for exon 4 (E4f; $5^{\prime}$-CCTGTGATGGAGTTGCTGGGCAGCGTGCTGAGTGG-3') and a reverse primer for exon 6 (E6r; 5'-AGCCCCCGGTGGTCCAATGCTGTT ACTCCGGAGG-3'). The resulting product was cloned into pCR2.1-TOPO (Invitrogen). The entire fragment spanning 3,539 base pairs was excised with the restriction enzyme PvuII (New England Biolabs) and ligated into the PmeI site of the targeting vector pOS (R. Thresher) with the Rapid DNA Ligation Kit (Roche). The $5^{\prime}$ arm of homology was obtained by PCR with intron 1 forward primer 1 (I1f) (5'-AGAGCACTTGCTGTTCATGCTGAGGTCGCACGACTTG) and exon 2 reverse primer (E2r) ( $5^{\prime}$-TTCACCTGCTCAGCTGGGGAGCCAACACTGT- $\left.3^{\prime}\right)$. The PCR product was cloned into pCR2.1-TOPO. A 1,902-base pair XbaI intron 1-exon 2 
fragment was subcloned into the NheI site in pOS. Both arms of homology were sequenced in their entirety for confirmation of the structure of the targeting construct. The targeting construct was transfected by electroporation into $129 \mathrm{~S} 6 / \mathrm{Sv}$ embryonic stem cells and cells were maintained for 14 under selection with the aminoglycoside G418 and gancyclovir. A clone identified as positive by Southern blot analysis and PCR was injected into C57BL/6 blastocysts. Chimeric male mice were crossed to either 129SV6 female mice or with C57BL/6 female mice. The resulting pups were screened by PCR for the presence of the mutated locus. DNA obtained from mouse tails was digested with XbaI, resolved by electrophoresis through a $0.8 \%$ TBE gel and transferred overnight to Zetaprobe nylon membranes (Bio-Rad). A 672-base pair probe of sequence upstream of exon 1was generated by PCR with forward primer $5^{\prime}$-TGCTCAGTCCCAGAATGGAGATCC- $3^{\prime}$ and reverse primer 5'-GCATCCTGCTCCGCCCATGCCATC-3', then was cloned into pCR2.1-TOPO (Invitrogen), excised with EcoRI, gel-purified and labeled with the Prime-a-Gene labeling system (Promega). The resulting fragments of 14.3 kilobases and 13.1 kilobases (representing the wild-type and targeted allele, respectively) were detected by Southern blot analysis of mouse genomic DNA digested with HindIII. Primers outside the region of homologous recombination were used for confirmation of the correct integration of the targeted allele (If1 (5'-AGCACTTGCTGTTCATGCTGAGGTCGCACGACTTG-3') and NeoPro, and E10r (5'-CATGTCACTGATGGAATTGC-3') and Neo3'-2 (5'-

TTCTATCGCCTTCTTGACGAG- $\left.3^{\prime}\right)$ ). Multiplex PCR was used for genotyping with primers specific for the $5^{\prime}$ arm of homology (Common $\left(5^{\prime}\right.$ ATTCACAACTTCTGGCTGCAAACAC- $\left.3^{\prime}\right)$, NeoPro (5' GGCCACACGCGTCACCTTAATATGCG-3') and wild-type reverse ( $5^{\prime}$ CTCAGAGTGCTTCGGTATCCTTGAC-3')). Heterozygous F1 progeny were interbred to obtain $\mathrm{NlrC}^{-1-}$ mice and were backcrossed for nine generations onto the C57BL/6 background. Mice were housed in a pathogen-free facility and maintained according to Institutional Animal Care and Use Committee guidelines. For LPS challenge, $4.5 \mathrm{mg}$ LPS per kg body weight was administered intra-peritoneally. The temperatures and weights of the mice were monitored three times a day for $10 \mathrm{~d}$. For time-point studies, mice were killed and serum and peritoneal lavage were collected. For all experiments, wild-type and $N 1 r 3^{-1-}$ mice were matched for age and sex. All peritoneal macrophages used were not preactivated with thioglycollate.

\section{Statistics}

Significance was determined with Student's $t$-test or one-way analysis of variance with Tukey's or Newman Kuhls post-test.

\section{Supplementary Material}

Refer to Web version on PubMed Central for supplementary material.

\section{Acknowledgments}

We thank S. Ghosh (Columbia University, New York) for hemagglutinin-tagged MyD88 and Flag-tagged IRAK; Y. Xiong (University of North Carolina) for plasmids encoding hemagglutinin-tagged K48-linked ubiquitin and K63-linked ubiquitin; A. Baldwin (University of North Carolina) for Flag-tagged IKKa; and R. Thresher (University of North Carolina) for the targeting vector pOS. Supported by the US National Institutes of Health (R37-AI029564, U54-AI057157, U19-AI1077437 and T32-AR007416).

\section{References}

1. Davis BK, Wen H, Ting JPY. The inflammasome NLRs in immunity, inflammation, and associated diseases. Annu Rev Immunol. 2010; 29:707-735. [PubMed: 21219188] 
2. Martinon F, Gaide O, Pétrilli V, Mayor A, Tschopp J. NALP inflammasomes: a central role in innate immunity. Semin Immunopathol. 2007; 29:213-229. [PubMed: 17703304]

3. Lamkanfi M, Dixit VM. Modulation of inflammasome pathways by bacterial and viral pathogens. J Immunol. 2011; 187:597-602. [PubMed: 21734079]

4. Davis BK, et al. Cutting Edge: NLRC5-dependent activation of the inflammasome. J Immunol. 2011; 186:1333-1337. [PubMed: 21191067]

5. Bruey JM, et al. PAN1/NALP2/PYPAF2, an inducible inflammatory mediator that regulates NF- $\kappa \mathrm{B}$ and caspase-1 activation in macrophages. J Biol Chem. 2004; 279:51897-51907. [PubMed: 15456791]

6. Khare S, et al. An NLRP7-containing inflammasome mediates recognition of microbial lipopeptides in human macrophages. Immunity. 2012; 36:464-476. [PubMed: 22361007]

7. Bauernfeind FG, et al. Cutting edge: NF- $\kappa$ B activating pattern recognition and cytokine receptors license NLRP3 inflammasome activation by regulating NLRP3 expression. J Immunol. 2009; 183:787-791. [PubMed: 19570822]

8. Chamaillard M, et al. An essential role for NOD1 in host recognition of bacterial peptidoglycan containing diaminopimelic acid. Nat Immunol. 2003; 4:702-707. [PubMed: 12796777]

9. Girardin SE. Nod1 detects a unique muropeptide from Gram-negative bacterial peptidoglycan. Science. 2003; 300:1584-1587. [PubMed: 12791997]

10. Girardin SE, et al. Peptidoglycan molecular requirements allowing detection by Nod1 and Nod2. J Biol Chem. 2003; 278:41702-41708. [PubMed: 12871942]

11. Inohara N, et al. Host recognition of bacterial muramyl dipeptide mediated through NOD2. Implications for Crohn's disease. J Biol Chem. 2003; 278:5509-5512. [PubMed: 12514169]

12. Bertrand MJM, et al. Cellular inhibitors of apoptosis cIAP1 and cIAP2 are required for innate immunity signaling by the pattern recognition receptors NOD1 and NOD2. Immunity. 2009; 30:789-801. [PubMed: 19464198]

13. Strober W, Murray PJ, Kitani A, Watanabe T. Signalling pathways and molecular interactions of NOD1 and NOD2. Nat Rev Immunol. 2005; 6:9-20. [PubMed: 16493424]

14. Abbott DW, et al. Coordinated regulation of Toll-like receptor and NOD2 signaling by K63-linked polyubiquitin chains. Mol Cell Biol. 2007; 27:6012-6025. [PubMed: 17562858]

15. Tao M, et al. ITCH K63-ubiquitinates the NOD2 binding protein, RIP2, to influence inflammatory signaling pathways. Curr Biol. 2009; 19:1255-1263. [PubMed: 19592251]

16. Ting JPY, Duncan JA, Lei Y. How the noninflammasome NLRs function in the innate immune system. Science. 2010; 327:286-290. [PubMed: 20075243]

17. Allen IC, et al. NLRP12 suppresses colon inflammation and tumorigenesis through the negative regulation of noncanonical NF- $\kappa$ B signaling. Immunity. 201210.1016/j.immuni.2012.03.012

18. Zaki MH, et al. The NOD-like receptor NLRP12 attenuates colon inflammation and tumorigenesis. Cancer Cell. 2011; 20:649-660. [PubMed: 22094258]

19. Moore CB, et al. NLRX1 is a regulator of mitochondrial antiviral immunity. Nature. 2008; 451:573-577. [PubMed: 18200010]

20. Allen IC, et al. NLRX1 protein attenuates inflammatory responses to infection by interfering with the RIG-I-MAVS and TRAF6-NF- $\times B$ signaling pathways. Immunity. 2011; 34:854-865. [PubMed: 21703540]

21. Xia X, et al. NLRX1 negatively regulates TLR-Induced NF- $\kappa B$ signaling by targeting TRAF6 and IKK. Immunity. 2011; 34:843-853. [PubMed: 21703539]

22. Benko S, Magalhaes JG, Philpott DJ, Girardin SE. NLRC5 limits the activation of inflammatory pathways. J Immunol. 2010; 185:1681-1691. [PubMed: 20610642]

23. Cui J, et al. NLRC5 negatively regulates the NF- $\kappa$ B and type I interferon signaling pathways. Cell. 2010; 141:483-496. [PubMed: 20434986]

24. Kumar H, et al. NLRC5 deficiency does not influence cytokine induction by virus and bacteria infections. J Immunol. 2011; 186:994-1000. [PubMed: 21148033]

25. Staehli F, et al. NLRC5 deficiency selectively impairs MHC class I-dependent lymphocyte killing by cytotoxic T cells. J Immunol. 2012; 188:3820-3828. [PubMed: 22412192] 
26. Tong Y, et al. Enhanced TLR-induced NF- $\kappa$ B signaling and type I interferon responses in NLRC5 deficient mice. Cell Res. 2012; 22:822-835. [PubMed: 22473004]

27. Rothe M, Sarma V, Dixit VM, Goeddel DV. TRAF2-mediated activation of NF- $\kappa$ B by TNF receptor 2 and CD40. Science. 1995; 269:1424-1427. [PubMed: 7544915]

28. Nakano H, et al. TRAF5, an activator of NF- $\kappa$ B and putative signal transducer for the lymphotoxin- $\beta$ receptor. J Biol Chem. 1996; 271:14661-14664. [PubMed: 8663299]

29. Hasegawa M, et al. A critical role of RICK/RIP2 polyubiquitination in Nod-induced NF- $\kappa B$ activation. EMBO J. 2008; 27:373-383. [PubMed: 18079694]

30. Devin A, et al. The distinct roles of TRAF2 and RIP in IKK activation by TNF-R1: TRAF2 recruits IKK to TNF-R1 while RIP mediates IKK activation. Immunity. 2000; 12:419-429. [PubMed: 10795740]

31. Conze DB, Wu CJ, Thomas JA, Landstrom A, Ashwell JD. Lys63-linked polyubiquitination of IRAK-1 is required for interleukin-1 receptor- and Toll-like receptor-mediated NF- $\kappa \mathrm{B}$ activation. Mol Cell Biol. 2008; 28:3538-3547. [PubMed: 18347055]

32. Lamothe B, et al. Site-specific Lys-63-linked tumor necrosis factor receptor-associated factor 6 auto-ubiquitination is a critical determinant of I $\mathrm{kB}$ kinase activation. J Biol Chem. 2006; 282:4102-4112. [PubMed: 17135271]

33. Sebban H, Yamaoka S, Courtois G. Posttranslational modifications of NEMO and its partners in NF- $\kappa B$ signaling. Trends Cell Biol. 2006; 16:569-577. [PubMed: 16987664]

34. Deng L, et al. Activation of the I $\mathrm{BB}$ kinase complex by TRAF6 requires a dimeric ubiquitinconjugating enzyme complex and a unique polyubiquitin chain. Cell. 2000; 103:351-361. [PubMed: 11057907]

35. Kishimoto K, Matsumoto K, Ninomiya-Tsuji J. TAK1 mitogen-activated protein kinase kinase kinase is activated by autophosphorylation within its activation loop. J Biol Chem. 2000; 275:7359-7364. [PubMed: 10702308]

36. Bishop GA. The multifaceted roles of TRAFs in the regulation of B-cell function. Nat Rev Immunol. 2004; 4:775-786. [PubMed: 15459669]

37. Lamothe B, et al. The RING domain and first zinc finger of TRAF6 coordinate signaling by interleukin-1, lipopolysaccharide, and RANKL. J Biol Chem. 2008; 283:24871-24880. [PubMed: 18617513]

38. Skaug B, Jiang X, Chen ZJ. The role of ubiquitin in NF- $\kappa$ B regulatory pathways. Annu Rev Biochem. 2009; 78:769-796. [PubMed: 19489733]

39. Trompouki E, et al. CYLD is a deubiquitinating enzyme that negatively regulates NF- $\kappa \mathrm{B}$ activation by TNFR family members. Nature. 2003; 424:793-796. [PubMed: 12917689]

40. Heyninck K. The cytokine-inducible zinc finger protein A20 inhibits IL-1-induced NF- $\kappa$ B activation at the level of TRAF6. FEBS Lett. 1999; 442:147-150. [PubMed: 9928991]

41. Boone DL, et al. The ubiquitin-modifying enzyme A20 is required for termination of Toll-like receptor responses. Nat Immunol. 2004; 5:1052-1060. [PubMed: 15334086]

42. Brummelkamp TR, Nijman SMB, Dirac AMG, Bernards R. Loss of the cylindromatosis tumour suppressor inhibits apoptosis by activating NF-кB. Nature. 2003; 424:797-801. [PubMed: 12917690]

43. Kovalenko A, et al. The tumour suppressor CYLD negatively regulates NF- $\kappa$ B signalling by deubiquitination. Nature. 2003; 424:801-805. [PubMed: 12917691]

44. Conti BJ. CATERPILLER 16.2 (CLR16.2), a novel NBD/LRR family member that negatively regulates T cell function. J Biol Chem. 2004; 280:18375-18385. [PubMed: 15705585]

45. Horie R, et al. A novel domain in the CD30 cytoplasmic tail mediates NFrB activation. Int Immunol. 1998; 10:203-210. [PubMed: 9533448]

46. Marinis JM, Homer CR, McDonald C, Abbott DW. A novel motif in the Crohn's disease susceptibility protein, NOD2, allows TRAF4 to down-regulate innate immune responses. J Biol Chem. 2010; 286:1938-1950. [PubMed: 21097508]

47. Ye H, Park YC, Kreishman M, Kieff E, Wu H. The structural basis for the recognition of diverse receptor sequences by TRAF2. Mol Cell. 1999; 4:321-330. [PubMed: 10518213] 
48. Coornaert B, Carpentier I, Beyaert R. A20: central gatekeeper in inflammation and immunity. J Biol Chem. 2008; 284:8217-8221. [PubMed: 19008218]

49. Sun SC. Deubiquitylation and regulation of the immune response. Nat Rev Immunol. 2008; 8:501511. [PubMed: 18535581]

50. Taxman DJ, et al. Criteria for effective design, construction, and gene knockdown by shRNA vectors. BMC Biotechnol. 2006; 6:7. [PubMed: 16433925] 

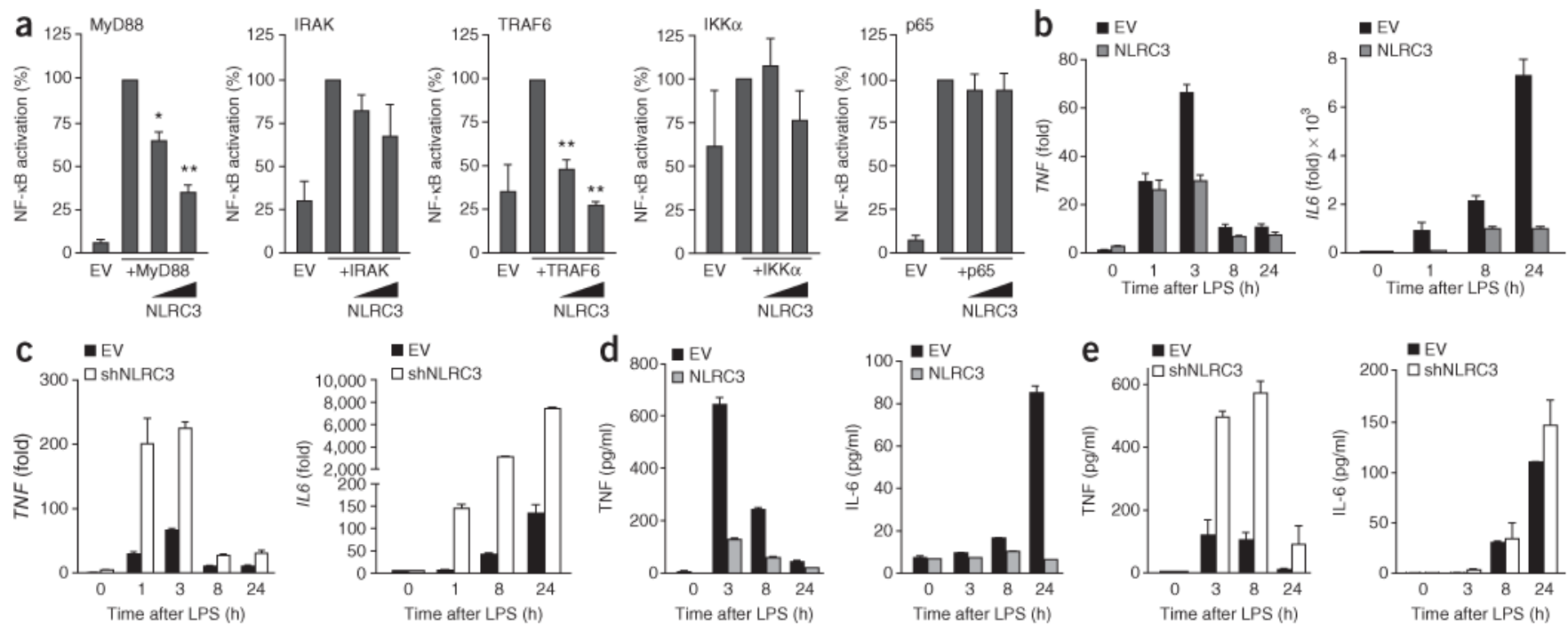

f Time after
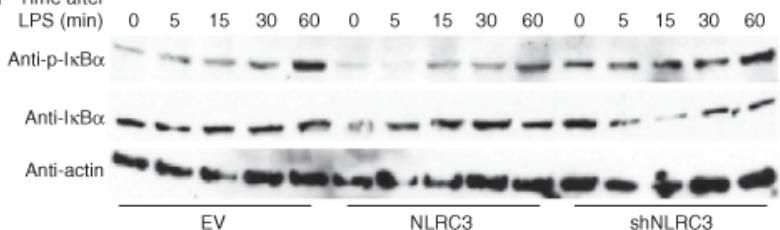

Figure 1.

NLRC3 inhibits MyD88- and TRAF6-dependent activation of NF- $\kappa$ B. (a) Luciferase activity in HEK293T cells transfected with a luciferase reporter vector driven by an NF- $\kappa \mathrm{B}-$ responsive promoter, plus empty vector (EV) or vector encoding MyD88, IRAK, TRAF6, IKKa or p65, and increasing concentrations (wedges) of a vector encoding NLRC3; results are presented relative to results obtained in the absence of NLRC3. $* P<0.001$ and $* * P<$ 0.0001 (Student's $t$-test). (b, c) Expression of TNF and IL6 in THP-1 cell lines stably transduced with retrovirus expressing empty vector or Flag-tagged NLRC3 (b) or empty vector or shNLRC3 (c) and stimulated with LPS; results were normalized to the expression of $A C T B$ (encoding $\beta$-actin) and are presented relative to those of untreated cells. (d, e) Production of TNF and IL- 6 by THP-1 cells transduced as in $\mathbf{b}(\mathbf{d})$ or $\mathbf{c}(\mathbf{e})$ and simulated with LPS. (f) Immunoblot analysis of total and phosphorylated (p-) I $\mathrm{BBa}$ in THP-1 cells transduced as in b, $\mathbf{c}$ and simulated with LPS; actin serves as a loading control throughout. Data are representative of at least three independent experiments (mean and s.e.m. in a-e). 
a

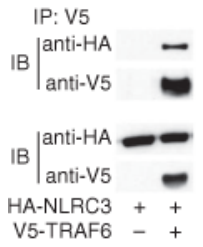

b

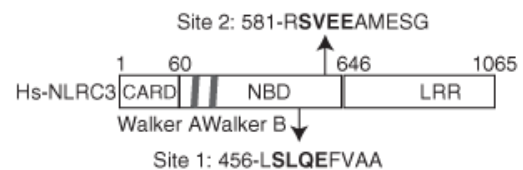

C

Homo sapiens Pan troglodyte. Canis lupus Bos taurus Mus musculus Rattus norvegicus Danio rerio

Homo sapiens Pan troglodytes Canis lupus Bos taurus Mus musculus Rattus norvegicus Danio rerio

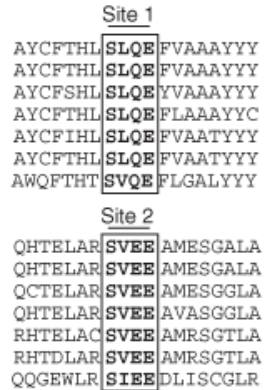

d

IP: V5-TRAF6

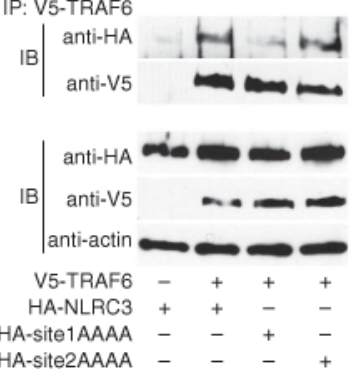

Figure 2.

NLRC3 inhibits NF- $\kappa$ B by association with TRAF6.

(a) Immunoprecipitation (IP) of V5-TRAF6 (with anti-V5 agarose) from HEK293T cells transiently transfected to express HA-NLRC3 plus V5-TRAF6 (+) or empty vector (-), followed by immunoblot analysis (IB) with antibody to hemagglutinin (anti-HA) or anti-V5.

(b) Major TRAF2-binding motifs (bold) in the NBD of NLRC3, identified by a bioinformatics search. Numbers above diagram and before sequences indicate amino acid positions. Hs-, Homo sapiens; CARD, caspase-recruitment domain; LRR, leucine-rich repeat; Walker A and Walker B, ATP-binding motifs. (c) Conservation across species of TRAF2-binding sites 1 and 2 from $\mathbf{b}$. (d) Immunoprecipitation of V5-TRAF6 from HEK293T cells transfected to express various combinations (below lanes) of V5-TRAF6 and hemagglutinin-tagged wild-type NLRC3 (HA-NLRC3) or NLRC3 mutants with replacement of amino acids in TRAF2-binding site 1 (HA-site1AAAA) or site 2 (HAsite2AAAA) with alanine, followed by immunoblot analysis with anti-hemagglutinin or anti-V5. Data are representative of at least three independent experiments. 
a

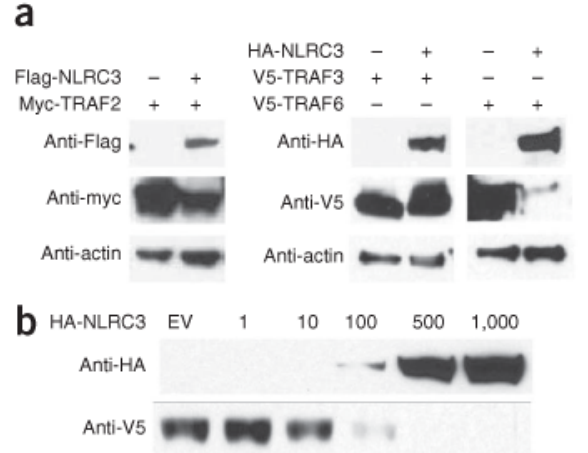

C

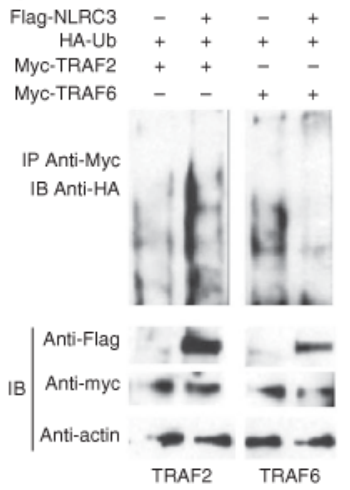

d

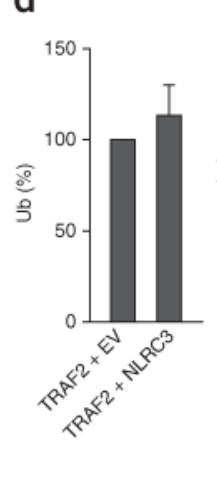

e

HA-K48 +--+-

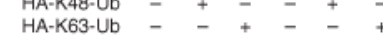

Flag-NLRC3 ---+++

V5-TRAF6 ++++++

IP V5-TRAF6

Anti-HA-Ub
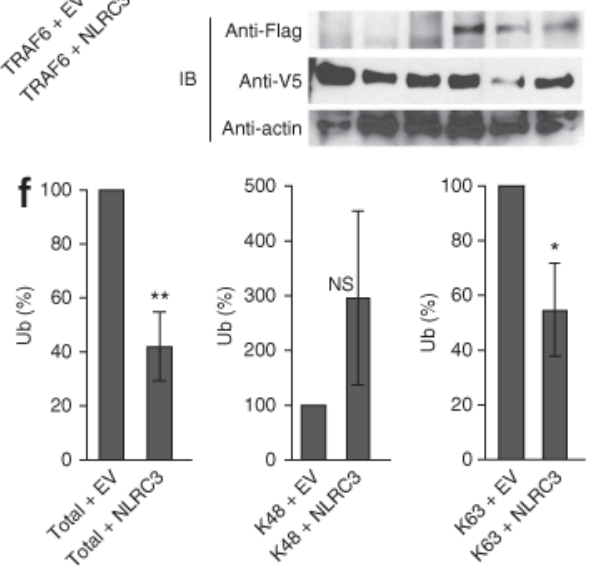

Figure 3.

NLRC3 promotes degradation of TRAF6 and prevents autoubiquitination of TRAF6. (a) Immunoblot analysis of HEK293T cells transfected to overexpress vectors encoding Myctagged TRAF2 or V5-tagged TRAF3 or TRAF6 with or without Flag- or HA-tagged NLRC3. (b) Immunoblot analysis of HEK293T cells transfected with vector encoding V5TRAF6 (2 $\mu \mathrm{g})$ and empty vector or increasing amounts (above lanes) of vector encoding HA-NLRC3. (c) Immunoblot analysis of the ubiquitination of TRAF6 and TRAF2 in HEK293T cells transfected to express Myc-tagged TRAF2 or TRAF6 and hemagglutinintagged ubiquitin (HA-Ub) in the presence $(+)$ or absence $(-)$ of vector encoding Flag-tagged NLRC3, assessed after (top) or without (below) immunoprecipitation with anti-Myc agarose. (d) Densitometry of the bands c, showing the ubiquitination of TRAF2 (left) and TRAF6 (right), presented relative to results obtained with cells transfected to express empty vector (instead of Flag-tagged NLRC3), set as $100 \%$. * $P=0.006$ (Student's $t$-test). (e) Immunoblot analysis of K48-linked and K63-linked ubiquitination of TRAF6 in cells transfected to express V5-TRAF6 with or without Flag-tagged NLRC3, plus plasmid encoding hemagglutinin-tagged total ubiquitin (HA-Ub), K48-linked ubiquitin (HA-K48$\mathrm{Ub}$ ) or K63-linked ubiquitin (HA-K63-Ub). (f) Densitometry of the bands e, showing the total (left), K48-linked (middle) and K63-linked (right) ubiquitination of TRAF6, normalized to total TRAF6 protein and presented as in $\mathbf{d} . * P=0.025$ and $* * P=0.005$ (Student's $t$-test). Data are representative of at least three experiments $(\mathbf{a}, \mathbf{b})$ or are from six $(\mathbf{c}, \mathbf{d})$ or five $(\mathbf{e}, \mathbf{f})$ independent experiments (average and s.e.m. in $\mathbf{d}$ and $\mathbf{f}$ ). 
a

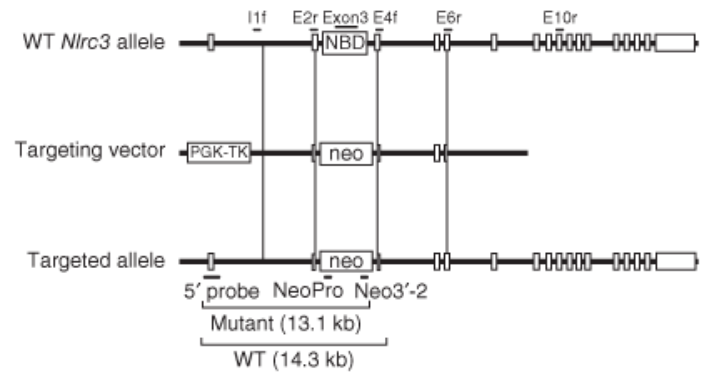

b

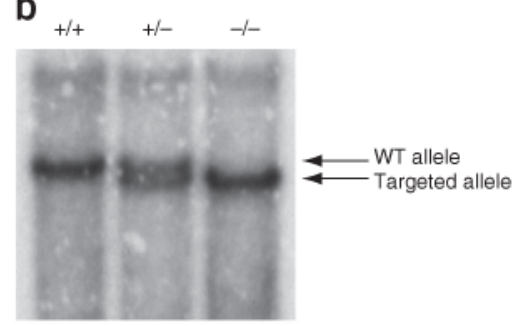

d

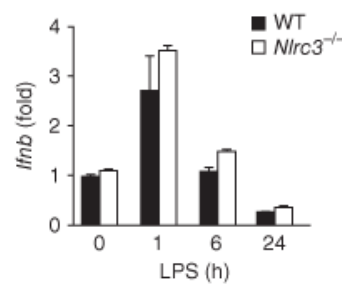

C
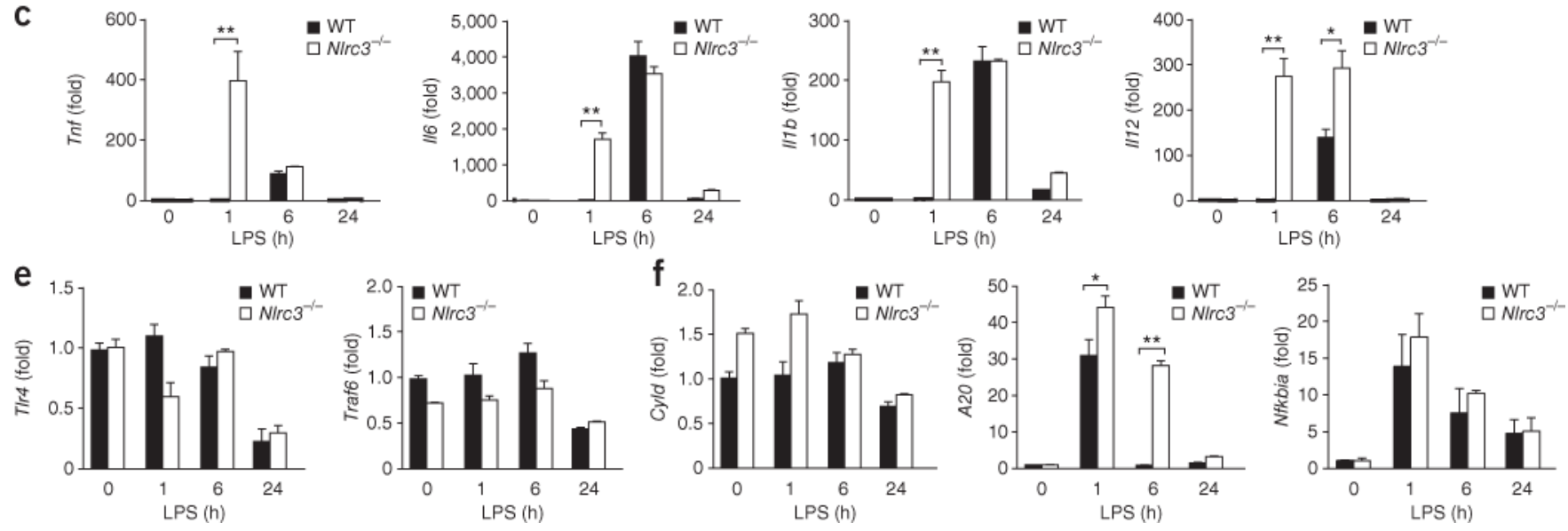

Figure 4.

$N \mathrm{NCS}^{-1-}$ macrophages produce more proinflammatory cytokines in response to TLR agonists. (a) Strategy used to disrupt the NIrc3 locus: homologous recombination of the targeting construct (middle) with endogenous wild-type (WT) NIrc3 (top) resulted in the replacement of genomic sequence spanning and including the final 103 base pairs of exon 2 through the first 35 base pairs of exon 4 with the gene encoding the selectable marker for geneticin (and neomycin) resistance (neo). Labels at top indicate primers: I 1f, intron 1 forward; E2r, exon 2 reverse; E4f, exon 4 forward; E6r, exon 6 reverse; E10r, exon 10 reverse. Below left ( $5^{\prime}$ probe), probe used in $\mathbf{b}$ that detects HindIII fragments (below diagram) generated from the wild-type locus and the targeted (Mutant) locus (size, in kilobases (kb), in parentheses). PGK-TK, gene encoding thymidine kinase linked to the promoter of the gene encoding phosphoglycerate kinase; NeoPro, neo reverse primer; Neo3'-2, neo forward primer (neo primers used to detect correct insertion of gene). (b) Southern blot analysis of DNA obtained from tail biopsies of mouse pups generated by the intercross of $\mathrm{NIrC}^{+1-}$ mice (probe in a). (c) Expression of Tnf, II6, IIIb and $I 112 \mathrm{mRNA}$ in wild-type and $\mathrm{NIrC}^{-1-}$ peritoneal macrophages stimulated with LPS, normalized to Gapdh (encoding glyceraldehyde phosphate dehydrogenase) and presented relative to that in untreated wild-type cells, set as 1. (d) Expression of the gene encoding interferon- $\beta$ (Ifnb) in wild-type and $\mathrm{NIrc}^{-/-}$peritoneal macrophages stimulated with LPS (presented as in $\mathbf{c}$ ). (e) Expression of TIr 4 and Traf6 in wild-type and $\mathrm{NIrc}^{-/-}$peritoneal macrophages stimulated with LPS (presented as in c). (f) Expression of Cyld, A20 and Nfkbia in wild-type and $N I r c 3^{-1-}$ macrophages stimulated with LPS (presented as in c). $* P<0.001$ and $* * P<0.0001$ (one-way analysis of variance with Tukey's post-test). Data are representative of one experiment (b) or three independent experiments (c-f; error bars, s.e.m.). 

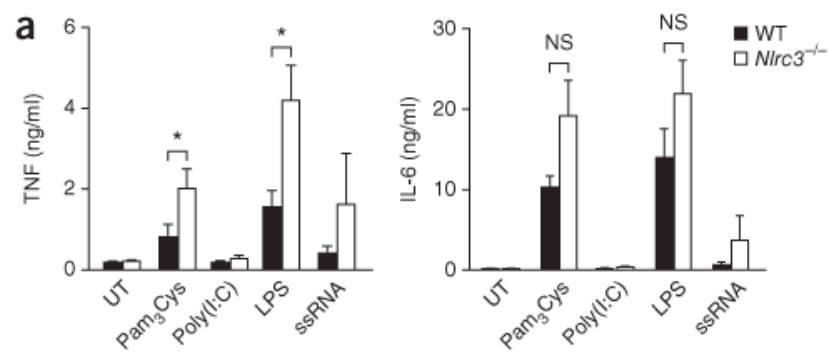

b
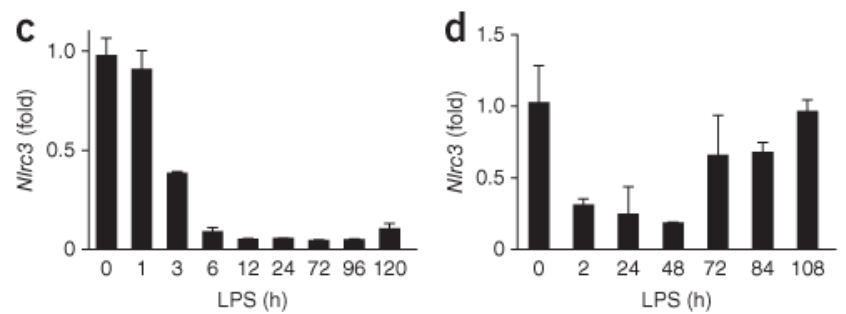

e

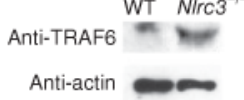
WT +-+
Nirc3 $^{-1-}-++$ LPS - ++

Anti-TRAF6 Anti-actin - - -

Figure 5.

NIrc $3^{-1-}$ macrophages produce more proinflammatory cytokines and TRAF6 in response to LPS. (a) Production of TNF and IL-6 by wild-type and NIrc $3^{-1}$ peritoneal macrophages left untreated (UT) or stimulated via the TLR. ssRNA, single-stranded RNA. NS, not significant $\left(P=0.11\right.$ (Pam ${ }_{3}$ Cys) or 0.079 (LPS)); $* P<0.05$ (Student's $t$-test). (b) Immunoblot analysis of TRAF6 in wild-type and $\mathrm{Nlrc}^{-1-}$ peritoneal macrophages. (c) Expression of NIrc3 in wildtype peritoneal macrophages stimulated for $8 \mathrm{~h}$ ex vivo with LPS, followed by replacement of medium and further culture for a total of $120 \mathrm{~h}$ (presented as in Fig. 4c). (d) Expression of NIrc3 in wild-type peritoneal cells after intraperitoneal challenge with LPS (presented as in Fig. 4c). (e) Immunoblot analysis of TRAF6 in wild-type and NIrc $3^{-/-}$ peritoneal macrophages left untreated (-) or stimulated for $24 \mathrm{~h}$ with LPS (+). Data are from at least four independent experiments (a; average and s.e.m.), three experiments (c; error bars, s.e.m.) or two experiments with at least four mice (d; average and s.e.m.) or are representative of at least three experiments (b) or three independent experiments (e). 
a

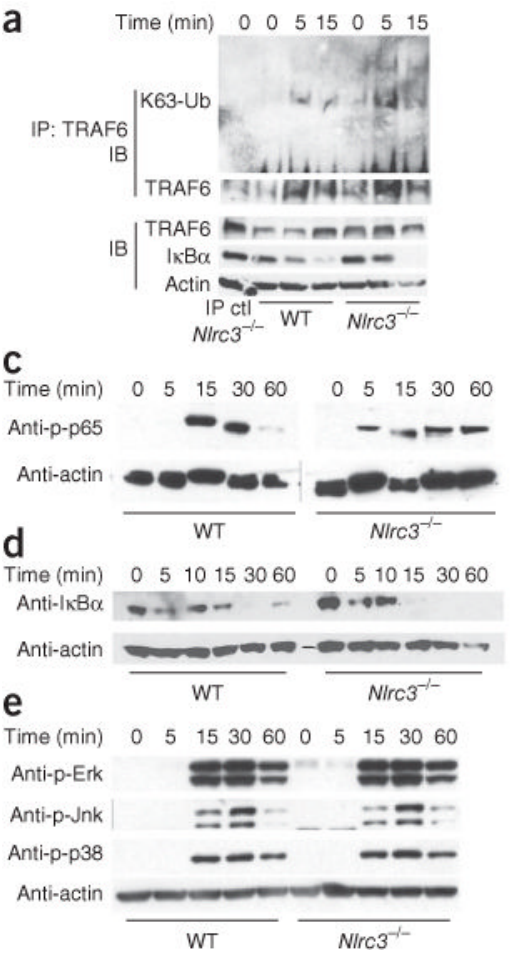

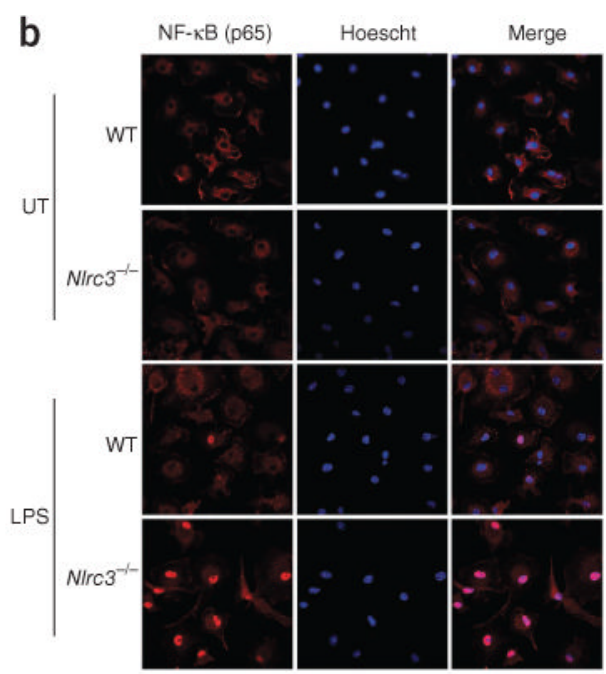

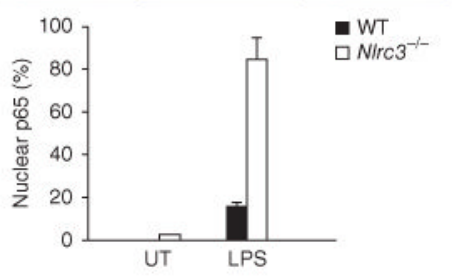

Figure 6.

NIrc $3^{-1-}$ macrophages have more K63-linked ubiquitination of TRAF6 and activation of NF- $\kappa$ B. (a) Immunoblot analysis of K63-linked ubiquitination (K63-Ub) of endogenous TRAF6 immunoprecipitated from wild-type and NIrc $3^{-/-}$bone marrow macrophages stimulated with LPS (top two bots), and immunoblot analysis of TRAF6 and I $\kappa \mathrm{Ba}$ in those same cells without immunoprecipitation (below). IP ctl NIrc ${ }^{-1-}$ (far left), unstimulated

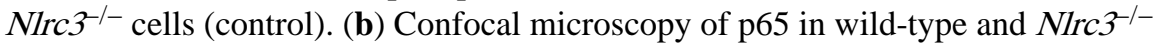
macrophages before (UT) and after (LPS) 15 min of stimulation with LPS. Original magnification, $\times 630$. Bottom, quantification of nuclear p65 in the images above (from at least three fields of view with at least 100 cells per sample). (c-e) Immunoblot analysis of phosphorylated p65 (c), the degradation of total I $\mathrm{KBa}(\mathbf{d})$ and phosphorylated Erk, Jnk and p38 (e) in wild-type and Nlrc ${ }^{-1}$ macrophages stimulated for various times (above lanes) with LPS. Data are representative of at least three independent experiments (error bars (b), s.e.m.). 

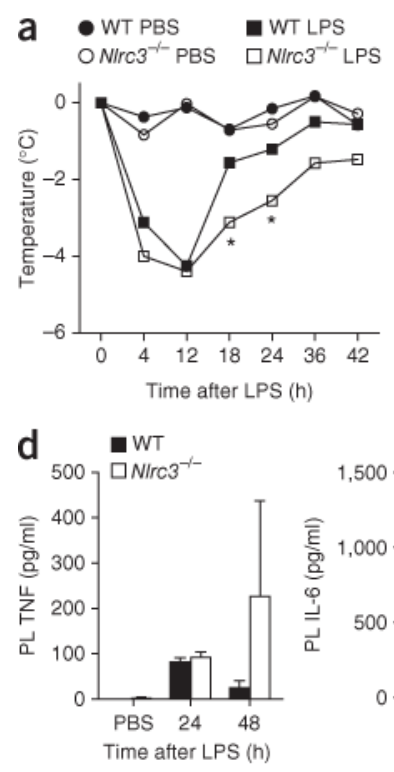

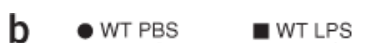
O Nirc3 ${ }^{-1-}$ PBS $\square \mathrm{NIrC}^{-1-}$ LPS
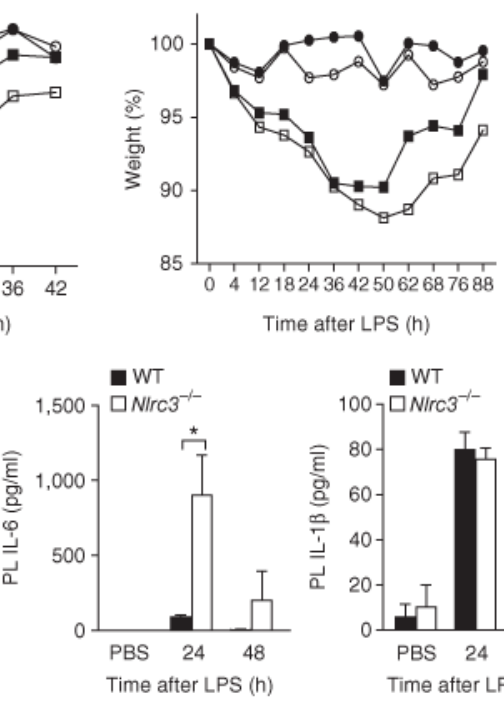

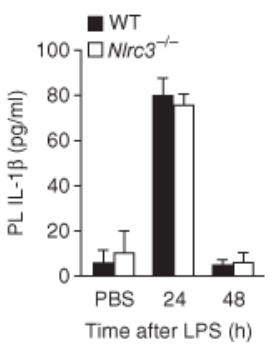

C
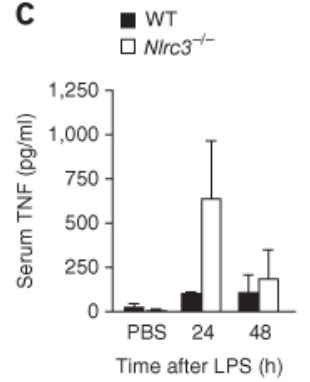

e

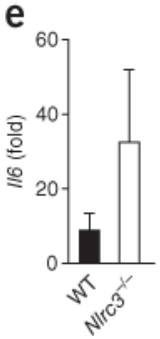

a WT
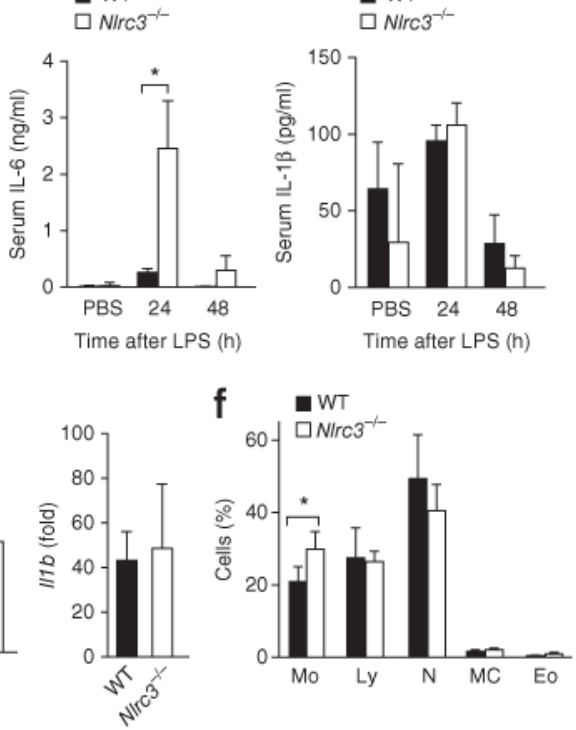

Figure 7.

NIrc $3^{-/-}$mice have a more severe reaction to sublethal challenge with LPS than do wildtype mice. (a, b) Change in body temperature (a) and weight (b) over time in wild-type and $\mathrm{Nlrc}^{-1-}$ mice challenged with LPS (4.5 mg per kg body weight). $* P<0.05$ (one-way analysis of variance with Newman-Kuels post-test). (c) TNF, IL- 6 and IL-1 $\beta$ in the serum of wild-type and $N I r c 3^{-/-}$mice at 24 or $48 \mathrm{~h}$ after LPS challenge as in a. $* P<0.01$ (Student's $t$-test). (d) TNF and IL-6 in the peritoneum (PL) of wild-type and NIrc $3^{-1-}$ mice at 24 or 48 $\mathrm{h}$ after LPS challenge as in a. $* P<0.005$ (Student's $t$-test). (e) Real-time PCR analysis of II6, Tnf and $I I 1 b$ in wild-type and NIrc ${ }^{-1-}$ peritoneal cells $24 \mathrm{~h}$ after LPS challenge as in a (presented as in Fig. 4c). (f) Monocytes (Mo), lymphocytes (Ly), neutrophils (N), mast cells (MC) and eosinophils (Eo) in peritoneal lavage fluid of wild-type and Nlrc3 $3^{-1-}$ mice $24 \mathrm{~h}$ after LPS challenge as in a, assessed by a differential Wright-Giemsa staining assay and presented relative to the total peritoneal cell population (in three fields). ${ }^{*} P<0.05$ (Student's $t$-test). Data are representative of at least four independent experiments $(\mathbf{a}, \mathbf{b}$; average of at least 12 mice per group), five experiments (c, $\mathbf{d}$; mean and s.e.m. of 9-16 mice), at least four experiments (e; error bars, s.e.m.) or three experiments (f; error bars, s.e.m.). 
Table 1

Minor and major TRAF2-binding motifs and TRAF6-binding motifs in NLR proteins

\begin{tabular}{|c|c|c|c|c|}
\hline NLR & NCBI reference sequence & TRAF2 (minor) & TRAF2 (major) & TRAF6 \\
\hline CIITA & NP_000237.2 & & 57 (AGEE); 674 (TLQE); 910 (AAEE); 1115 (SVQE) & $\begin{array}{l}29 \text { (PLEGGY); } 337 \\
\text { (PVEQFY) }\end{array}$ \\
\hline NAIP & NP_004527.2 & & $\begin{array}{c}232 \text { (SSEE); } 276 \text { (AYEE); } 448 \text { (PVQE); } 746 \text { (AFQE); } \\
1068 \text { (AEQE); } 1219 \text { (PDEE) }\end{array}$ & \\
\hline Nod1 & AAD43922.1 & & 479 (TQEE) & \\
\hline Nod2 & NP_071445.1 & & 106 (AAQE); 1024 (SLEE) & \\
\hline NLRC3 & NP_849172.2 & & 457 (SLQE); 582 (SVEE) & \\
\hline NLRC4 & AAH31555.1 & & 445 (SFQE); 541 (TEQE) & \\
\hline NLRC5 & NP_115582.3 & & $\begin{array}{c}516 \text { (SLQE); } 1210 \text { (SNEE); } 1429 \text { (PRQE); } 1607 \\
\text { (SLEE) }\end{array}$ & 273 (PSELLF) \\
\hline NLRP1 & AAH51787.1 & 994 (PTEGLD) & 169 (PSQE); 1047 (SHEE) & 1247 (PEEVTF) \\
\hline NLRP2 & AAH39269.1 & & 612 (SQEE) & \\
\hline NLRP3 & NP_004886.3 & & 112 (SIEE); 179 (SQQE); 524 (TFQE); 1020 (TLQE) & 689 (PKEEEE) \\
\hline NLRP4 & NP_604393.2 & & 51 (SREE); 232 (SFEE); 971 (TAEE) & 284 (PKELRD) \\
\hline NLRP5 & NP_703148.4 & & 244 (AEEE); 706 (SFQE) & \\
\hline NLRP6 & NP_612202.1 & & $\begin{array}{c}25 \text { (ALEE); } 158 \text { (APEE); } 165 \text { (PAEE); } 501 \text { (SFQE); } \\
606 \text { (PEEE); } 857 \text { (SLQE) }\end{array}$ & $\begin{array}{l}165 \text { (PAEEPE); } 606 \\
\text { (PEEEEE); } 618 \\
\text { (PLELLY) }\end{array}$ \\
\hline NLRP7 & AAI09126.1 & & 505 (SGEE); 578 (SQEE) & \\
\hline NLRP8 & NP_789781.2 & & 49 (SHEE); 497 (AGEE); 509 (TFQE) & \\
\hline NLRP9 & NP_789790.2 & & 88 (AQEE); 499 (STEE); 964 (SVEE) & \\
\hline NLRP10 & NP_789791.1 & & 143 (PEQE); 257 (PFEE); 316 (SEEE); 612 (PKEE) & \\
\hline NLRP11 & NP_659444.2 & & 48 (TKEE) & \\
\hline NLRP12 & NP_653288.1 & & 202 (PDEE); 516 (SFQE) & \\
\hline NLRP13 & NP_789780.2 & 38 (PQQLMD) & $\begin{array}{c}117 \text { (PTQE); } 140 \text { (PNQE); } 163 \text { (PNQE); } 295 \text { (PIEE); } \\
538 \text { (SFQE); } 645 \text { (SQEE) }\end{array}$ & \\
\hline NLRP14 & NP_789792.1 & & 91 (AKEE); 243 (PIEE); 267 (AFEE); 334 (AREE) & 67 (PGEKAW) \\
\hline NLRX1 & AAI10891.1 & 672 (PSELLD) & 94 (PREE); 459 (TEEE); 496 (AMQE) & $\begin{array}{l}262 \text { (PEEPQE); } 611 \\
\text { (PDEPPE) }\end{array}$ \\
\hline
\end{tabular}

Sequences of TRAF-binding motifs in various NLR proteins; numbers before sequences (in one-letter code) correspond to first amino acid of motif. P-X-(Q/E)-X-X-D is the minor TRAF2-binding motif (and is the TRAF6-binding motif if third residue is Glu (E)); (P/S/A/T)-X-(Q/E)-E is the major TRAF2-binding motif; and P-X-E-X-X-aromatic (F/W/Y)-acidic (D/E)) is the TRAF6-binding motif (where ' $\mathrm{X}$ ' indicates any amino acid and ' $\%$ indicates 'or'). The minor TRAF2-binding motifs for NLRP1 and NLRX1 may also be TRAF6-binding motifs. 